\title{
Weak Solution of a Singular Semilinear Elliptic Equation in a Bounded Domain
}

By

Robert DALMASSO*

\begin{abstract}
We study the singular semilinear elliptic equation $\Delta u+f(., u)=0$ in $\mathcal{D}^{\prime}(\Omega)$, where $\Omega \subset \mathbb{R}^{n}(n \geq 1)$ is a bounded domain of class $C^{1,1} . f: \Omega \times(0, \infty) \rightarrow[0, \infty)$ is such that $f(., u) \in L^{1}(\Omega)$ for $u>0$ and $u \rightarrow f(x, u)$ is continuous and nonincreasing for a.e. $x$ in $\Omega$. We assume that there exists a subset $\Omega^{\prime} \subset \Omega$ with positive measure such that $f(x, u)>0$ for $x \in \Omega^{\prime}$ and $u>0$ and that $\int_{\Omega} f(x, c d(x, \partial \Omega)) d x<\infty$ for all $c>0$. Then we show that there exists a unique solution $u$ in $W_{0}^{1,1}(\Omega)$ such that $\Delta u \in L^{1}(\Omega), u>0$ a.e. in $\Omega$.
\end{abstract}

\section{$\S 1 . \quad$ Introduction}

Let $\Omega$ be a sufficiently smooth (e.g. of class $C^{1,1}$ ) bounded domain in $\mathbb{R}^{n}$ $(n \geq 1)$. We consider the singular boundary value problem

$$
\begin{aligned}
& \Delta u+f(., u)=0 \quad \text { in } \quad \mathcal{D}^{\prime}(\Omega), \\
& u \in W_{0}^{1,1}(\Omega), f(., u(.)) \in L^{1}(\Omega),
\end{aligned}
$$

where $f$ satisfies the following conditions:

(H1) $f: \Omega \times(0, \infty) \rightarrow[0, \infty)$. For all $u>0, x \rightarrow f(x, u)$ is in $L^{1}(\Omega)$, and $u \rightarrow f(x, u)$ is continuous and nonincreasing for a.e. $x$ in $\Omega$;

(H2) There exists $\Omega^{\prime} \subset \Omega$ with positive measure such that $f(x, u)>0$ for $x \in \Omega^{\prime}$ and $u>0$;

Communicated by H. Okamoto. Received July 12, 2004.

2000 Mathematics Subject Classification(s): 35J25, 35J60.

*Laboratoire LMC-IMAG, Equipe EDP, Tour IRMA, BP 53, F-38041 Grenoble Cedex 9, France.

e-mail: Robert.Dalmasso@imag.fr

(C) 2005 Research Institute for Mathematical Sciences, Kyoto University. All rights reserved. 
(H3) For all $c>0$

$$
\int_{\Omega} f(x, c d(x, \partial \Omega)) d x<+\infty .
$$

This kind of singularity has been considered by several authors, particularly the case where

$$
f(x, u)=p(x) u^{-\lambda},
$$

$\lambda>0[4,5,6,9,10,11$, and their references $]$.

Lazer and McKenna [10] for instance established the existence and uniqueness of a positive $u \in C^{2}(\Omega) \cap C(\bar{\Omega})$ satisfying

$$
\begin{aligned}
\Delta u+p(x) u^{-\lambda}=0 & \text { in } \Omega, \\
u=0 & \text { on } \partial \Omega,
\end{aligned}
$$

when $p$ is Hölder-continuous and strictly positive in $\bar{\Omega}$.

Del Pino [6] proved that if $p$ is a bounded, nonnegative measurable function which is positive on a set of positive measure, then (1.3)-(1.4) has a unique positive weak solution in the sense that $u \in C^{1, \alpha}(\Omega) \cap C(\bar{\Omega})$ satisfies (1.4), $u>0$ in $\Omega$ and

$$
\int_{\Omega} \nabla u \nabla \varphi=\int_{\Omega} p u^{-\lambda} \varphi
$$

for all $\varphi \in C_{c}^{\infty}(\Omega)$.

Lair and Shaker [9] considered the case

$$
f(x, u)=p(x) g(u),
$$

under the following assumptions:

(A0) $p \in L^{2}(\Omega)$ is nontrivial and nonnegative;

(A1) $g^{\prime}(s) \leq 0$;

(A2) $g(s)>0$ if $s>0$;

(A3) $\int_{0}^{\varepsilon} g(s) d s<\infty$ for some $\varepsilon>0$.

They established the existence of a unique weak solution in the sense that $u \in H_{0}^{1}(\Omega)$ satisfies

$$
\int_{\Omega}(\nabla u \nabla v-p(x) g(u) v) d x=0 \quad \forall v \in H_{0}^{1}(\Omega) .
$$

Notice that, when $g(u)=u^{-\lambda}$, conditions (A1) and (A3) imply that $0 \leq$ $\lambda<1$. 
Finally Mâagli and Zribi [11] treated the general case $f(x, u)$. However their assumptions are different from ours and they lead them to the existence of a weak solution in $C(\bar{\Omega})$.

Our purpose is to give a general existence and uniqueness result under sufficiently weak conditions. We shall prove the following theorem.

Theorem 1. Let $\Omega \subset \mathbb{R}^{n}(n \geq 1)$ be a bounded domain of class $C^{1,1}$ and let $f: \Omega \times(0, \infty) \rightarrow[0, \infty)$ satisfy $(\mathrm{H} 1)-(\mathrm{H} 3)$. Then problem $(1.1)-(1.2)$ has a unique solution.

\section{§2. Proof of Theorem 1}

1) Uniqueness of the solution. We shall need the following lemma ([7, Lemma 3]).

Lemma 1. Let $p \in C^{1}(\mathbb{R}, \mathbb{R}) \cap L^{\infty}(\mathbb{R})$ be a nondecreasing function satisfying $p(0)=0$. For $u \in W_{0}^{1,1}(\Omega)$ such that $\Delta u \in L^{1}(\Omega)$ we have

$$
\int \Delta u \cdot p(u) \leq 0
$$

Let $u_{1}, u_{2}$ be two solutions of problem (1.1)-(1.2). Let $u=u_{1}-u_{2}$. By (H1) we have $u \Delta u \geq 0$ a.e. in $\Omega$. Now let $p \in C^{1}(\mathbb{R}) \cap L^{\infty}(\mathbb{R})$ be a strictly increasing function satisfying $p(0)=0$. Then $p(u) \Delta u \geq 0$ a.e. in $\Omega$. Using Lemma 1 we deduce that $p(u) \Delta u=0$ a.e. in $\Omega$ and therefore $\Delta u=0$ a.e. in $\Omega$. Since $u \in W_{0}^{1,1}(\Omega)$, this implies that $u=0$ a.e. in $\Omega$.

2) Existence of a solution. We first recall the following result ([1, Lemme 2.8]).

Lemma 2. Let $u \in W_{0}^{1,1}(\Omega)$ be such that $\Delta u \geq 0$ in $\mathcal{D}^{\prime}(\Omega)$. Then $u \leq 0$ a.e. in $\Omega$.

In the sequel $\mathbb{N}^{\star}$ denotes the set of positive integers.

Lemma 3. Let $j \in \mathbb{N}^{\star}$. There exists a unique $u_{j} \in W_{0}^{1,1}(\Omega)$ such that $f\left(., u_{j}+\frac{1}{j}\right) \in L^{1}(\Omega), u_{j} \geq 0$ a.e. in $\Omega$ and $\Delta u_{j}+f\left(., u_{j}+\frac{1}{j}\right)=0$ in $\mathcal{D}^{\prime}(\Omega)$.

Proof. Define

$$
\beta_{j}(x, u)=f\left(x, \frac{1}{j}\right)-f\left(x, u+\frac{1}{j}\right), \quad x \in \Omega, u \geq 0 .
$$


and

$$
\beta_{j}(x, u)=0, \quad x \in \Omega, u \leq 0 .
$$

Then we have:

- For all $u \in \mathbb{R}, x \rightarrow \beta_{j}(x, u)$ is in $L^{1}(\Omega)$;

- $\mathbb{R} \ni u \rightarrow \beta_{j}(x, u)$ is continuous and nondecreasing for a.e. $x$ in $\Omega$;

- $\beta_{j}(x, 0)=0$ for a.e. $x$ in $\Omega$.

Since $f\left(., \frac{1}{j}\right) \in L^{1}(\Omega)$ Theorem 3 in [7] implies the existence of a unique $u_{j} \in W_{0}^{1,1}(\Omega)$ satisfying $\beta_{j}\left(., u_{j}\right) \in L^{1}(\Omega)$ and

$$
-\Delta u_{j}+\beta_{j}\left(., u_{j}\right)=f\left(., \frac{1}{j}\right) \quad \text { in } \quad \mathcal{D}^{\prime}(\Omega) .
$$

Since $\Delta u_{j} \leq 0$ in $\mathcal{D}^{\prime}(\Omega)$, Lemma 2 implies that $u_{j} \geq 0$ a.e. in $\Omega$. Therefore we have $f\left(., u_{j}+\frac{1}{j}\right) \in L^{1}(\Omega)$ and

$$
\Delta u_{j}+f\left(., u_{j}+\frac{1}{j}\right)=0 \quad \text { in } \quad \mathcal{D}^{\prime}(\Omega),
$$

and the lemma is proved.

Lemma 4. For every $j \in \mathbb{N}^{\star}$ there exists $a_{j}>0$ such that

$$
u_{j}(x) \geq a_{j} d(x, \partial \Omega), \quad \text { for a.e. } x \in \Omega \text {. }
$$

Proof. For $\varepsilon>0$ we set $\Omega_{\varepsilon}=\{x \in \Omega ; d(x, \partial \Omega)>\varepsilon\}$. Clearly (H2) implies that, for every $j \in \mathbb{N}^{\star}$, there exist $\varepsilon_{j}>0$ and $M_{j}>0$ such that the function $\tilde{f}_{j}$ defined by

$$
\tilde{f}_{j}(x)=\min \left(f\left(x, u_{j}(x)+\frac{1}{j}\right), M_{j}\right) \mathbf{1}_{\Omega_{\varepsilon_{j}}}(x), \quad x \in \Omega,
$$

satisfies $\tilde{f}_{j} \not \equiv 0$. Let $v_{j}$ be the solution of the following boundary value problem

$$
\begin{aligned}
\Delta v_{j}+\tilde{f}_{j}=0 & \text { in } \quad \Omega, \\
v_{j}=0 & \text { on } \quad \partial \Omega .
\end{aligned}
$$

It is well-known (see [8]) that, for $1<p<\infty, v_{j} \in C^{1}(\bar{\Omega}) \cap W^{2, p}(\Omega)$. We have $\Delta\left(u_{j}-v_{j}\right) \leq 0$ in $\mathcal{D}^{\prime}(\Omega)$, hence by Lemma $2 u_{j} \geq v_{j}$ a.e. in $\Omega$. Now the boundary point version of the Strong Maximum Principle for weak solutions ([12], Theorem 2) implies that there exists $a_{j}>0$ such that $v_{j}(x) \geq a_{j} d(x, \partial \Omega)$ for $x \in \Omega$ and the lemma follows. 
Lemma 5. For every $j \in \mathbb{N}^{\star}$ we have $u_{j}+\frac{1}{j} \geq u_{j+1}+\frac{1}{j+1}$ a.e. in $\Omega$.

Proof. Let $u=\left(u_{j+1}+\frac{1}{j+1}\right)-\left(u_{j}+\frac{1}{j}\right)$. Using a variant of Kato's inequality (see [3] Lemma A1 in the Appendix) we deduce that

$$
\Delta u^{+} \geq 0 \quad \text { in } \mathcal{D}^{\prime}(\Omega)
$$

$u^{+} \in W_{0}^{1,1}(\Omega)$ (see [1, Lemme 2.7]). Therefore Lemma 2 implies that $u \leq 0$ a.e. in $\Omega$ and the lemma is proved.

Lemma 6. For every $j \in \mathbb{N}^{\star}$ we have $u_{j} \leq u_{j+1}$ a.e. in $\Omega$.

Proof. Using (H1) and Lemma 5 we get

$$
\Delta\left(u_{j+1}-u_{j}\right)=f\left(., u_{j}+\frac{1}{j}\right)-f\left(., u_{j+1}+\frac{1}{j+1}\right) \leq 0 \quad \text { a.e. in } \Omega,
$$

and we conclude with the help of Lemma 2 .

Now we define

$$
c_{j}=\int_{\Omega} f\left(x, u_{j}(x)+\frac{1}{j}\right) d x, \quad j \in \mathbb{N}^{\star} .
$$

Using Lemma 4 and Lemma 6 we can write

$$
c_{j} \leq \int_{\Omega} f\left(x, a_{1} d(x, \partial \Omega)\right) d x, \quad \forall j \in \mathbb{N}^{\star} .
$$

Therefore

$$
\sup _{j \in \mathbb{N}^{\star}} c_{j}<\infty
$$

Now we can prove the existence. By (H1) and Lemma $5 j \rightarrow f\left(., u_{j}+\frac{1}{j}\right)$ is nondecreasing. (3.1) and the Beppo Levi theorem for monotonic sequences imply that there exists $g \in L^{1}(\Omega)$ such that

$$
f\left(., u_{j}+\frac{1}{j}\right) \rightarrow g \quad \text { in } L^{1}(\Omega) \quad \text { as } j \rightarrow \infty .
$$

We have the following estimate $[2$, Theorem 8$]$ : for $1 \leq q<N /(N-1)$ there exists $M_{q}>0$ such that

$$
\left\|u_{j}\right\|_{W^{1, q}(\Omega)} \leq M_{q}\left\|\Delta u_{j}\right\|_{L^{1}(\Omega)} \quad \forall j \in \mathbb{N}^{\star}
$$


Therefore there exists $u \in W_{0}^{1,1}(\Omega)$ such that $u_{j} \rightarrow u$ in $W_{0}^{1,1}(\Omega)$. By Lemma 6 and the Fischer-Riesz theorem $u_{j} \rightarrow u$ a.e. in $\Omega$. Lemma 4 and Lemma 6 imply that $u>0$ a.e. in $\Omega$. Clearly we have $g=f(., u)$ and $\Delta u+f(., u)=0$ in $\mathcal{D}^{\prime}(\Omega)$. The proof is complete.

\section{References}

[1] Benilan, P. and Boulaamayel, B., Sous solutions d'équations elliptiques dans $L^{1}$, Potential Analysis, 10 (1999), 215-241.

[2] Brezis, H. and Strauss, W. A., Semilinear second order elliptic equations in $L^{1}$, J. Math. Soc. Japan, 25 (1973), 565-590.

[3] Brezis, H., Semilinear equations in $\mathbb{R}^{N}$ without condition at infinity, Appl. Math. Optim., 12 (1984), 271-282.

[4] Crandall, M. G., Rabinowitz, P. H. and Tartar, L., On a Dirichlet problem with a singular nonlinearity, Comm. Partial Differential Equations, 2 (1977), 193-222.

[5] Dalmasso, R., On singular nonlinear elliptic problems of second and fourth orders, Bull. Sc. Math., 116 (1992), 95-110.

[6] del Pino, M. A., A global estimate for the gradient in a singular elliptic boundary value problem, Proc. R. Soc. Edinburgh Sect. A, 122 (1992), 341-352.

[7] Gallouet, Th. and Morel, J. M., Resolution of a semilinear equation in $L^{1}$, Proc. R. Soc. Edinburgh Sect. A, 96 (1984), 275-288.

[8] Gilbarg, D. and Trudinger, N. S., Elliptic partial differential equations of second order, 2nd edn, Springer, Berlin, 1983.

[9] Lair, A. V. and Shaker, A. W., Classical and weak solutions of a singular semilinear elliptic problem, J. Math. Anal. Appl., 211 (1997), 371-385.

[10] Lazer, A. C. and McKenna, P. J., On a singular nonlinear elliptic boundary value problem, Proc. Amer. Math. Soc., 111 (1991), 721-730.

[11] Mâagli, H. and Zribi, M., Existence and estimates of solutions for singular nonlinear elliptic problems. J. Math. Anal. Appl., 263 (2001), 522-542.

[12] Vázquez, J. L., A strong maximum principle for some quasilinear elliptic equations, Appl. Math. Optim., 12 (1984), 191-202. 